\title{
An Algorithm: Optimal Homotopy Asymptotic Method for Solutions of Systems of Second-Order Boundary Value Problems
}

\author{
Muhammad Rafiq Mufti, ${ }^{1}$ Muhammad Imran Qureshi, ${ }^{1}$ Salem Alkhalaf, ${ }^{2}$ and S. Iqbal ${ }^{3}$ \\ ${ }^{1}$ Department of Computer Sciences, COMSATS Institute of Information Technology, Vehari, Pakistan \\ ${ }^{2}$ College of Sciences \& Arts, Computer Science Department, Qassim University, Alrass City, Saudi Arabia \\ ${ }^{3}$ Department of Informatics \& Systems, School of Systems and Technology, University of Management and Technology, Lahore, Pakistan
}

Correspondence should be addressed to Muhammad Imran Qureshi; imranqureshi18@gmail.com

Received 13 June 2016; Revised 26 October 2016; Accepted 22 November 2016; Published 3 January 2017

Academic Editor: Maria L. Gandarias

Copyright (C) 2017 Muhammad Rafiq Mufti et al. This is an open access article distributed under the Creative Commons Attribution License, which permits unrestricted use, distribution, and reproduction in any medium, provided the original work is properly cited.

\begin{abstract}
Optimal homotopy asymptotic method (OHAM) is proposed to solve linear and nonlinear systems of second-order boundary value problems. OHAM yields exact solutions in just single iteration depending upon the choice of selecting some part of or complete forcing function. Otherwise, it delivers numerical solutions in excellent agreement with exact solutions. Moreover, this procedure does not entail any discretization, linearization, or small perturbations and therefore reduces the computations a lot. Some examples are presented to establish the strength and applicability of this method. The results reveal that the method is very effective, straightforward, and simple to handle systems of boundary value problems.
\end{abstract}

\section{Introduction}

In this presentation, we study the nonlinear system of secondorder differential equations [1-3] of the subsequent type:

$$
\begin{aligned}
u^{\prime \prime} & +a_{1}(x) u^{\prime}+a_{2}(x) u+a_{3}(x) v^{\prime \prime}+a_{4}(x) v^{\prime} \\
& +a_{5}(x) v+G_{1}(x, u, v)=f_{1}(x), \\
v^{\prime \prime} & +b_{1}(x) v^{\prime}+b_{2}(x) v+b_{3}(x) u^{\prime \prime}+b_{4}(x) u^{\prime} \\
& +b_{5}(x) u+G_{2}(x, u, v)=f_{2}(x),
\end{aligned}
$$

with boundary conditions:

$$
\begin{aligned}
& u(0)=u(1)=0, \\
& v(0)=v(1)=0,
\end{aligned}
$$

where $0 \leq x \leq 1$ and $G_{1}$ and $G_{2}$ are nonlinear functions of $u$ and $v$, respectively. Also, $f_{1}$ and $f_{2}$ are known forcing functions of the system and $a_{i}(x), b_{i}(x)$ for $i=1,2, \ldots, 5$ are given continuous functions. In [1], the analytical solution of the above problem is illustrated in the form of series under the assumption that the solution is unique. In $[4,5]$, Sinccollocation and the Chebyshev finite difference methods, respectively, are used to solve the same systems. A numerical method based on the cubic B-spline scaling functions is proposed in [6] to find the solutions of (1)-(2). Also, in [2], the variational iteration method is presented to elucidate problem (1)-(2). He's homotopy perturbation method (HPM) is proposed to solve the same nonlinear systems of secondorder boundary value problems [3].

In the present work, optimal homotopy asymptotic method (OHAM) is extended to demonstrate the solutions of systems of boundary value problems (BVP). This method finds the exact solutions of system (1) having boundary conditions (2) for the choice of selecting some part of or complete forcing function; otherwise, it produces numerical solutions in excellent agreement with exact solutions. In recent years, the application of OHAM in linear and nonlinear problems has been developed by scientists and engineers, because this method deforms the difficult problems under study into simple problems, which are easy to solve. The OHAM was proposed first by the Romanian researcher Marinca et al. [7] in 2008. The advantage of OHAM is integrated convergence criteria similar to HAM but flexible to a greater extent in implementation. Marinca et al. [8-10] and Iqbal et al. [1115 ] in a series of papers have established validity, usefulness, 
simplification, and consistency of the method and acquired reliable solutions of currently significant applications in science and technology.

The organization of this presentation is as follows: In Section 2, extended algorithm of OHAM is illustrated for our subsequent progress. As a result, systems of simple differential equations are formed and the exact solutions of the considered problems are introduced. In Section 3, some examples of linear and nonlinear systems are answered by the extended algorithm, results to clarify the method with existing exact results. Section 4 ends this paper with a brief conclusion.

\section{OHAM Formulation (an Algorithm) for Systems of BVP}

According to the optimal homotopy asymptotic method [718 ], the following is the extended formulation for system of boundary value problems:

(a) Write the governing system of differential equations as follows:

$$
A(x) U^{\prime \prime}(x)=F(x)-G(x, U)-B(x) U^{\prime}-C(x) U,
$$

where $U^{\prime \prime}(x)=\left[u_{1}^{\prime \prime}(x), u_{2}^{\prime \prime}(x), \ldots, u_{n}^{\prime \prime}(x)\right]$ is an unknown vector function to be determined. $A(x)=\left[a_{i j}(x)\right], B(x)=$ $\left[b_{i j}(x)\right]$, and $C(x)=\left[c_{i j}(x)\right], i, j=1,2, \ldots, n$, are known matrices having functions

$$
\begin{aligned}
U^{\prime}(x) & =\left[u_{1}^{\prime}(x)+u_{2}^{\prime}(x), \ldots, u_{n}^{\prime}(x)\right], \\
U(x) & =\left[u_{1}(x), u_{2}(x), \ldots, u_{n}(x)\right], \\
F(x) & =\left[f_{1}(x), f_{2}(x), \ldots, f_{n}(x)\right],
\end{aligned}
$$

which are given vector functions.

$G(x, U)=\left[g_{1}(x, U), g_{2}(x, U), \ldots, g_{n}(x, U)\right]$ are given nonlinear vector functions, and $x \in \Omega$ is domain. Now, consider the $i$ th equation of (3) to be of the following form:

$$
\begin{gathered}
\mathrm{eq}^{i \text { th }} \equiv \sum_{j=1}^{n} a_{i j} u_{j}^{\prime \prime}+g_{i}(x, U(x))+\sum_{j=1}^{n} b_{i j} u_{j}^{\prime} \\
+\sum_{j=1}^{n} c_{i j} u_{j}-f_{i}(x)=0
\end{gathered}
$$

for $i=1,2, \ldots, n, a_{i i}=1$, and $\mathrm{eq}^{i \text { th }}$ is $i$ th equation of any such system.

(b) Construct an optimal homotopy in an unconventional way which satisfies the following equation:

$$
(1-p)\left\{a_{i i} u_{i}^{\prime \prime}+f_{i 1}(x)\right\}-H\left(x ; p, C_{i}\right)\left\{\mathrm{eq}^{i \mathrm{th}}\right\}=0,
$$

where $p \in[0,1]$ is an embedding parameter increasing monotonically from zero to unity as $u_{i}(x ; 0)=u_{i, 0}$ is continuously deformed to $u_{i}(x ; 1)=u_{i}(x), f_{i}(x)=f_{i 1}(x)+$ $f_{i 2}(x)$, and $H\left(x ; p, C_{i}\right)=p K_{1}\left(x, C_{i}\right)+p^{2} K_{2}\left(x, C_{i}\right)+\cdots+$ $p^{m} K_{m}\left(x, C_{i}\right)$ is a nonzero auxiliary function for $p \neq 0$, where $C_{1}, C_{2}, \ldots$ are constants to be determined, ensuring the fast convergence. The functions $K_{1}, K_{2}, K_{3}, \ldots, K_{m}$ reduce to constants for simple problems and for complicated problems these functions depend on $x$ and $C_{i}$. The choices of functions $K_{m}\left(x, C_{i}\right)$ might be exponential, polynomial, and soon. It is very significant to choose these functions, since the convergence of the solution very much depends on these functions. The auxiliary function $H\left(x ; p, C_{i}\right)$ provides us with a simple way to adjust and control the convergence. It also increases the accuracy of the results and effectiveness of the method $[12,17,18]$.

(c) Expand (5) in the manner of Taylor's series expansion about $p$ to get the solution of the following form:

$$
\begin{array}{r}
u_{i}\left(x ; p, C_{1}, C_{2}, \ldots\right)=u_{i 0}(x)+\sum_{l} u_{i l}\left(x ; C_{1}, C_{2}, \ldots\right) p^{l}, \\
i=1,2, \ldots, n .
\end{array}
$$

The convergence of the series equation (7) depends upon the auxiliary constants $C_{1}, C_{2}, \ldots$, and if it is convergent at $p=1$, then one has

$$
u_{i}\left(x ; C_{i}\right)=u_{i 0}(x)+\sum_{l} u_{i l}\left(x ; C_{i}\right), \quad i=1,2, \ldots, n .
$$

(d) Substituting (8) into (6) takes the following form:

$$
\begin{aligned}
& (1-p)\left\{a_{i i}\left(u_{i 0}^{\prime \prime}+p u_{i 1}^{\prime \prime}+p^{2} u_{i 2}^{\prime \prime}+\cdots+f_{i 1}(x)\right)\right\} \\
& -H(p)\left\{\sum_{j=1}^{n} a_{i j}\left(u_{i 0}^{\prime \prime}+p u_{i 1}^{\prime \prime}+p^{2} u_{i 2}^{\prime \prime}+\cdots\right)\right. \\
& +g_{i}(x, U(x))+\sum_{j=1}^{n} b_{i j}\left(u_{i 0}^{\prime}+p u_{i 1}^{\prime}+p^{2} u_{i 2}^{\prime}+\cdots\right) \\
& \left.+\sum_{j=1}^{n} c_{i j}\left(u_{i 0}+p u_{i 1}+p^{2} u_{i 2}+\cdots\right)-f_{i}(x)\right\}=0 .
\end{aligned}
$$

(e) Compare the coefficients of the like powers of $p$; one can get the different order problems. Solution of these simple problems can be obtained easily. After substitution of these results in (8), one can get $m$ th order approximation as

$$
\begin{aligned}
u_{i}^{m} & \left(x ; C_{1}, C_{2}, \ldots, C_{m}\right) \\
& =u_{i 0}(x)+\sum_{l=1}^{m} u_{i l}\left(x ; C_{1}, C_{2}, \ldots, C_{l}\right),
\end{aligned}
$$

$$
\text { where } i=1,2, \ldots, m \text {. }
$$

(f) Substituting (10) into (5) results in the residual $R_{i}\left(x ; C_{1}, C_{2}, \ldots, C_{m}\right)$. If $R_{i}=0$ for $i=1,2, \ldots, n$ then $u_{i}^{m}(x)$ will be the exact solution of the system. Generally, it does not happen, especially in nonlinear problems.

(g) Construct the functional for the determinations of optimal values of auxiliary constants, $C_{i}, i=1,2, \ldots, m$, as

$$
J_{i}\left(C_{1}, C_{2}, \ldots, C_{m}\right)=\int_{\Omega} R_{i}^{2}\left(C_{1}, C_{2}, \ldots, C_{m}\right) d x
$$

where $i=1,2, \ldots, n$. 
Optimal values of auxiliary constants for solutions of system can be determined by minimizing functional (11) as follows:

$$
\frac{\partial J_{i}}{\partial C_{1}}=\frac{\partial J_{i}}{\partial C_{2}}=\cdots=\frac{\partial J_{i}}{\partial C_{m}}=0, \quad i=1,2, \ldots, n
$$

Note. To determine the solutions of systems of boundary value problems, the choice of $f_{i 1}(x)$ from $f_{i}(x)$ of the systems is very important. It has been observed in this article that exact solutions may be obtained by the proper choice of $f_{i 1}(x)$. Example 3 has been discussed for different $f_{i 1}(x)$.

\section{Explanatory Examples}

This section is devoted to explanatory examples. The extended method presented in this paper is applied to the three systems of boundary value problems. These problems are chosen such that they have exact solutions.

Example 1. A linear system of second-order boundary value problems is as follows [2, 3]:

$$
\begin{gathered}
u^{\prime \prime}(x)+x u(x)+x v(x)=2, \\
v^{\prime \prime}(x)+2 x v(x)+2 x u(x)=-2,
\end{gathered}
$$

$$
0 \leq x \leq 1
$$

subject to the boundary conditions

$$
\begin{aligned}
& u(0)=u(1)=0, \\
& v(0)=v(1)=0 .
\end{aligned}
$$

The exact solutions are $u(x)=x^{2}-x$ and $v(x)=x-x^{2}$. A series of problems are generated by OHAM formulation presented in the previous section. The expressions for zerothorder, first-order, and second-order problems of the system and their solutions are given below:

$$
\begin{aligned}
& \frac{d^{2} u_{0}(x)}{d x^{2}}-2=0, \\
& u_{0}(0)=0, \\
& u_{0}(1)=0, \\
& \Downarrow \\
& u_{0}(x)=x^{2}-x ; \\
& \frac{d^{2} v_{0}(x)}{d x^{2}}+2=0, \\
& v_{0}(0)=0, \\
& v_{0}(1)=0, \\
& \Downarrow \\
& v_{0}(x)=x-x^{2} ;
\end{aligned}
$$

$$
\begin{aligned}
& \frac{d^{2} u_{1}(x)}{d x^{2}}-\left(1+C_{1}\right) \frac{d^{2} u_{0}(x)}{d x^{2}}-x C_{1}\left(u_{0}(x)+v_{0}(x)\right) \\
& +2\left(1+C_{1}\right)=0 \\
& u_{1}(0)=0 \text {, } \\
& u_{1}(1)=0 \text {, } \\
& \Downarrow \\
& u_{1}(x)=0 \text {; } \\
& \frac{d^{2} v_{1}(x)}{d x^{2}}-\left(1+C_{1}\right) \frac{d^{2} v_{0}(x)}{d x^{2}} \\
& -2 x C_{1}\left(u_{0}(x)+v_{0}(x)\right)-2\left(1+C_{1}\right)=0, \\
& v_{1}(0)=0 \text {, } \\
& v_{1}(1)=0 \text {, } \\
& \Downarrow \\
& v_{1}(x)=0 ; \\
& \frac{d^{2} u_{2}(x)}{d x^{2}}-\left(1+C_{1}\right) \frac{d^{2} u_{1}(x)}{d x^{2}}-C_{2} \frac{d^{2} u_{0}(x)}{d x^{2}} \\
& -x C_{1}\left(u_{1}(x)+v_{1}(x)\right)-x C_{2}\left(u_{0}(x)+v_{0}(x)\right) \\
& +2 C_{2}=0 \text {, } \\
& u_{2}(0)=0 \text {, } \\
& u_{2}(1)=0 \text {, } \\
& \Downarrow \\
& u_{2}(x)=0 \text {; } \\
& \frac{d^{2} v_{2}(x)}{d x^{2}}-\left(1+C_{1}\right) \frac{d^{2} v_{1}(x)}{d x^{2}}-C_{2} \frac{d^{2} v_{0}(x)}{d x^{2}} \\
& -2 x C_{1}\left(u_{1}(x)+v_{1}(x)\right)-2 x C_{2}\left(u_{0}(x)+v_{0}(x)\right) \\
& -2 C_{2}=0 \text {, } \\
& v_{2}(0)=0 \text {, } \\
& v_{2}(1)=0 \text {, } \\
& \Downarrow \\
& v_{2}(x)=0 .
\end{aligned}
$$

By using (10), (e) part of the OHAM algorithm, solutions of the system are

$$
\begin{aligned}
& u(x)=u_{0}(x)+u_{1}(x)+u_{2}(x)+\cdots, \Longrightarrow \\
& u(x)=x^{2}-x ; \\
& v(x)=v_{0}(x)+v_{1}(x)+v_{2}(x)+\cdots, \Longrightarrow \\
& v(x)=x-x^{2}
\end{aligned}
$$


Equations (16) show the exact solutions of the system given in Example 1. In this system, (f) and (g) parts of the algorithms are not used.

Example 2. Consider the following system $[2,3]$ :

$$
\begin{aligned}
u^{\prime \prime}(x)+(2 x-1) u^{\prime}(x)+\cos (\pi x) v^{\prime}(x) & =f_{1}(x), \\
v^{\prime \prime}(x)+x u(x) & =f_{2}(x),
\end{aligned}
$$$$
0 \leq x \leq 1,
$$

subject to the following boundary conditions:

$$
\begin{aligned}
& u(0)=u(1)=0, \\
& v(0)=v(1)=0,
\end{aligned}
$$

where

$$
\begin{aligned}
f_{1}(x)= & -\pi^{2} \sin (\pi x)+(2 x-1) \pi \cos (\pi x) \\
& +(2 x-) \cos (\pi x), \\
f_{2}(x)= & 2+x \sin (\pi x) .
\end{aligned}
$$

According to algorithm (b) (see (6)) of OHAM presented in previous section, $f_{11}(x)=-\pi^{2} \sin (\pi x)$ and $f_{21}(x)=2$, since $f_{1}(x)=f_{11}(x)+f_{12}(x)$ and $f_{2}(x)=f_{21}(x)+f_{22}(x)$. The exact solutions of system in Example 2 are $u(x)=\sin (\pi x)$ and $v(x)=x^{2}-x$. According to OHAM formulation, the expressions for zeroth-order, first-order, and second-order problems of the system and their solutions are given below:

$$
\begin{aligned}
& \frac{d^{2} u_{0}(x)}{d x^{2}}+\pi^{2} \sin (\pi x)=0, \\
& u_{0}(0)=0 \\
& u_{0}(1)=0 \text {, } \\
& \Downarrow \\
& u_{0}(x)=\sin (\pi x) \\
& \frac{d^{2} v_{0}(x)}{d x^{2}}-2=0, \\
& v_{0}(0)=0 \text {, } \\
& v_{0}(1)=0 \text {, } \\
& \Downarrow \\
& v_{0}(x)=x^{2}-x ; \\
& \frac{d^{2} u_{1}(x)}{d x^{2}}-\left(1+C_{1}\right) \frac{d^{2} u_{0}(x)}{d x^{2}}-\cos (\pi x) C_{1} v_{0}^{\prime}(x) \\
& +(1-2 x) C_{1} u_{0}^{\prime}(x)-\left(1+C_{1}\right) \pi^{2} \sin (\pi x) \\
& +C_{1}(2 x-1) \pi \cos (\pi x) \\
& +C_{1}(2 x-1) \cos (\pi x)=0 \text {, }
\end{aligned}
$$

$$
\begin{aligned}
& u_{1}(0)=0 \text {, } \\
& u_{1}(1)=0 \text {, } \\
& \Downarrow \\
& u_{1}(x)=0 \text {; } \\
& \frac{d^{2} v_{1}(x)}{d x^{2}}-\left(1+C_{1}\right) \frac{d^{2} v_{0}(x)}{d x^{2}}-x C_{1} u_{0}(x) \\
& +2\left(1+C_{1}\right)+C_{1} x \sin (\pi x)=0, \\
& v_{1}(0)=0 \text {, } \\
& v_{1}(1)=0 \text {, } \\
& \Downarrow \\
& v_{1}(x)=0 \text {; } \\
& \frac{d^{2} u_{2}(x)}{d x^{2}}-\left(1+C_{1}\right) \frac{d^{2} u_{1}(x)}{d x^{2}}-C_{2} \frac{d^{2} u_{0}(x)}{d x^{2}} \\
& -\cos (\pi x) C_{1} v_{1}^{\prime}(x)-\cos (\pi x) C_{2} v_{0}^{\prime}(x) \\
& +(1-2 x) C_{1} u_{1}^{\prime}(x)+(1-2 x) C_{2} u_{0}^{\prime}(x) \\
& -C_{2} \pi^{2} \sin (\pi x)+C_{2}(2 x-1) \pi \cos (\pi x) \\
& +C_{2}(2 x-1) \cos (\pi x)=0, \\
& u_{2}(0)=0 \text {, } \\
& u_{2}(1)=0 \text {, } \\
& \Downarrow \\
& u_{2}(x)=0 \text {; } \\
& \frac{d^{2} v_{2}(x)}{d x^{2}}-\left(1+C_{1}\right) \frac{d^{2} v_{1}(x)}{d x^{2}}-C_{2} \frac{d^{2} v_{0}(x)}{d x^{2}} \\
& -x C_{1} u_{1}(x)-x C_{2} u_{0}(x)+2 C_{2} \\
& +C_{2} x \sin (\pi x)=0 \text {, } \\
& v_{2}(0)=0 \text {, } \\
& v_{2}(1)=0 \text {, } \\
& \Downarrow \\
& v_{2}(x)=0 \text {. }
\end{aligned}
$$

By using (10), (e) part of the OHAM algorithm, solutions of the system are

$$
\begin{aligned}
& u(x)=u_{0}(x)+u_{1}(x)+u_{2}(x)+\cdots, \Longrightarrow \\
& u(x)=\sin (\pi x) ; \\
& v(x)=v_{0}(x)+v_{1}(x)+v_{2}(x)+\cdots, \Longrightarrow \\
& v(x)=x^{2}-x .
\end{aligned}
$$


Equations (21) show the exact solutions of the system given in Example 2.

Example 3. Consider the following nonlinear system $[2,3]$ :

$$
\begin{array}{r}
u^{\prime \prime}(x)-x v^{\prime}(x)+u(x)=f_{1}(x), \\
v^{\prime \prime}(x)+x u^{\prime}(x)+u(x) v(x)=f_{2}(x),
\end{array}
$$

$$
0 \leq x \leq 1,
$$

subject to the following boundary conditions:

$$
\begin{gathered}
u(0)=u(1)=0, \\
v(0)=v(1)=0,
\end{gathered}
$$

where $f_{1}(x)=x^{3}-2 x^{2}+6 x$ and $f_{2}(x)=x^{5}-x^{4}+2 x^{3}+$ $x^{2}-x+2$. The exact solutions of system in Example 3 are $u(x)=x^{3}-x$ and $v(x)=x^{2}-x$. As discussed in Note at the end of previous section, Example 3 has been discussed here for different choices of $f_{i 1}(x)$.

1st Choice $\left(f_{11}(x)=6 x\right.$ and $\left.f_{21}(x)=2\right)$. According to the algorithm (b) (see (6)), of OHAM, $f_{11}(x)=6 x$ and $f_{21}(x)=2$, since $f_{1}(x)=f_{11}(x)+f_{12}(x)$ and $f_{2}(x)=$ $f_{21}(x)+f_{22}(x)$. The expressions for zeroth-order, first-order, and second-order problems of the system and their solutions are given below:

$$
\begin{aligned}
& \frac{d^{2} u_{0}(x)}{d x^{2}}-6 x=0, \\
& u_{0}(0)=0, \\
& u_{0}(1)=0, \\
& \Downarrow \\
& u_{0}(x)=x^{3}-x ; \\
& \frac{d^{2} v_{0}(x)}{d x^{2}}-2=0, \\
& v_{0}(0)=0, \\
& v_{0}(1)=0, \\
& \Downarrow \\
& v_{0}(x)=x^{2}-x ; \\
& \frac{d^{2} u_{1}(x)}{d x^{2}}-\left(1+C_{1}\right) \frac{d^{2} u_{0}(x)}{d x^{2}}+x C_{1} v_{0}^{\prime}(x)-C_{1} u_{0}(x) \\
& \quad+C_{1}\left(x^{3}-2 x^{2}+6 x\right)+6 x=0, \\
& u_{1}(0)=0, \\
& u_{1}(1)=0,
\end{aligned}
$$

$\Downarrow$

$$
\begin{aligned}
& u_{1}(x)=0 \text {; } \\
& \frac{d^{2} v_{1}(x)}{d x^{2}}-\left(1+C_{1}\right) \frac{d^{2} v_{0}(x)}{d x^{2}}-x C_{1} u_{0}^{\prime}(x) \\
& -C_{1} u_{0}(x) v_{0}(x) \\
& +2 C_{1}\left(x^{5}-x^{4}+2 x^{3}+x^{2}-x+2\right)=0 \text {, } \\
& v_{1}(0)=0 \text {, } \\
& v_{1}(1)=0 \text {, } \\
& \Downarrow \\
& v_{1}(x)=0 ; \\
& \begin{array}{l}
\frac{d^{2} u_{2}(x)}{d x^{2}}-\left(1+C_{1}\right) \frac{d^{2} u_{1}(x)}{d x^{2}}-C_{2} \frac{d^{2} u_{0}(x)}{d x^{2}} \\
+x C_{1} v_{1}^{\prime}(x)+x C_{2} v_{0}^{\prime}(x)-C_{1} u_{1}(x)-C_{2} u_{0}(x) \\
\quad+C_{2}\left(x^{3}-2 x^{2}+6 x\right)=0
\end{array} \\
& u_{2}(0)=0 \text {, } \\
& u_{2}(1)=0 \text {, } \\
& \Downarrow \\
& u_{2}(x)=0 \text {; } \\
& \frac{d^{2} v_{2}(x)}{d x^{2}}-\left(1+C_{1}\right) \frac{d^{2} v_{1}(x)}{d x^{2}}-C_{2} \frac{d^{2} v_{0}(x)}{d x^{2}} \\
& -x C_{1} u_{1}^{\prime}(x)-x C_{2} u_{0}^{\prime}(x)-C_{1} u_{0}(x) v_{1}(x) \\
& -C_{1} u_{1}(x) v_{0}(x)-C_{2} u_{0}(x) v_{0}(x) \\
& +C_{2}\left(x^{5}-x^{4}+2 x^{3}+x^{2}-x+2\right)=0, \\
& v_{2}(0)=0 \text {, } \\
& v_{2}(1)=0 \text {, } \\
& \Downarrow \\
& v_{2}(x)=0 .
\end{aligned}
$$

By using (10), (e) part of the OHAM algorithm, solutions of the system are

$$
\begin{aligned}
& u(x)=u_{0}(x)+u_{1}(x)+u_{2}(x)+\cdots, \Longrightarrow \\
& u(x)=x^{3}-x \\
& v(x)=v_{0}(x)+v_{1}(x)+v_{2}(x)+\cdots, \Longrightarrow \\
& v(x)=x^{2}-x
\end{aligned}
$$

Equations (25) show the exact solutions of the system given in Example 3. 
2nd Choice $\left(f_{11}(x)=f_{1}(x)\right.$ and $\left.f_{21}(x)=f_{2}(x)\right)$. The expressions for zeroth-order, first-order, and second-order problems of the system and their solutions are given below:

$$
\begin{aligned}
& \frac{d^{2} u_{0}(x)}{d x^{2}}-x^{3}+2 x^{2}-6 x=0, \\
& u_{0}(0)=0 \text {, } \\
& u_{0}(1)=0 \text {, } \\
& \Downarrow \\
& u_{0}(x)=\frac{1}{60}\left(-53 x+60 x^{3}-10 x^{4}+3 x^{5}\right) \\
& \frac{d^{2} v_{0}(x)}{d x^{2}}-x^{5}+x^{4}-2 x^{3}-x^{2}+x-2=0, \\
& v_{0}(0)=0 \text {, } \\
& v_{0}(1)=0 \text {, } \\
& \Downarrow \\
& v_{0}(x)=\frac{1}{420}\left(-423 x+420 x^{2}-70 x^{3}+35 x^{4}+42 x^{5}-14 x^{6}+10 x^{7}\right) \\
& \frac{d^{2} u_{1}(x)}{d x^{2}}-\left(1+C_{11}\right) \frac{d^{2} u_{0}(x)}{d x^{2}}+x C_{11} v_{0}^{\prime}(x)-C_{11} u_{0}(x)+\left(1+C_{11}\right)\left(x^{3}-2 x^{2}+6 x\right)=0, \\
& u_{1}(0)=0 \text {, } \\
& u_{1}(1)=0 \text {, } \\
& \Downarrow \\
& u_{1}(x)=\frac{x\left(1469+312 x^{2}-2520 x^{3}+1134 x^{4}-252 x^{5}-162 x^{6}+54 x^{7}-35 x^{8}\right) C_{11}}{15120} \\
& \frac{d^{2} v_{1}(x)}{d x^{2}}-\left(1+C_{21}\right) \frac{d^{2} v_{0}(x)}{d x^{2}}-x C_{21} u_{0}^{\prime}(x)-C_{21} u_{0}(x) v_{0}(x)+\left(1+C_{21}\right)\left(x^{5}-x^{4}+2 x^{3}+x^{2}-x+2\right)=0 \\
& v_{1}(0)=0 \text {, } \\
& v_{1}(1)=0 \text {, } \\
& \Downarrow \\
& v_{1}(x)=\frac{1}{1816214400} x\left(-15492027-267387120 x^{2}+134648514 x^{3}+192216024 x^{4}-92420328 x^{5}+58129500 x^{6}\right. \\
& \left.-15308865 x^{7}+4806802 x^{8}+1145144 x^{9}-756756 x^{10}+472836 x^{11}-65604 x^{12}+11880 x^{13}\right) C_{21} ; \\
& \frac{d^{2} u_{2}(x)}{d x^{2}}-\left(1+C_{11}\right) \frac{d^{2} u_{1}(x)}{d x^{2}}-C_{12} \frac{d^{2} u_{0}(x)}{d x^{2}}+x C_{11} v_{1}^{\prime}(x)+x C_{12} v_{0}^{\prime}(x)-C_{11} u_{1}(x)-C_{12} u_{0}(x)+C_{12}\left(x^{3}-2 x^{2}\right. \\
& +6 x)=0 \\
& u_{2}(0)=0 \text {, } \\
& u_{2}(1)=0 \text {, }
\end{aligned}
$$




$$
\begin{aligned}
& u_{2}(x)=-\frac{1}{54486432000} x\left(5 4 6 0 \left(-839543-367510 x^{2}+1663200 x^{3}-758736 x^{4}+221760 x^{5}+89100 x^{6}-32670 x^{7}\right.\right. \\
& \left.+24585 x^{8}-396 x^{9}+210 x^{10}\right) C_{11}^{2}+3603600\left(-1469-312 x^{2}+2520 x^{3}-1134 x^{4}+252 x^{5}+162 x^{6}-54 x^{7}+35 x^{8}\right) \\
& \cdot C_{12}+C_{11}\left(3603600\left(-1469-312 x^{2}+2520 x^{3}-1134 x^{4}+252 x^{5}+162 x^{6}-54 x^{7}+35 x^{8}\right)+(210332406\right. \\
& -77460135 x^{2}-1203242040 x^{4}+538594056 x^{5}+686485800 x^{6}-297065340 x^{7}+169544375 x^{8}-40823640 x^{9} \\
& \left.\left.\left.+11798514 x^{10}+2602600 x^{11}-1600830 x^{12}+935280 x^{13}-121836 x^{14}+20790 x^{15}\right) C_{21}\right)\right) ; \\
& \frac{d^{2} v_{2}(x)}{d x^{2}}-\left(1+C_{21}\right) \frac{d^{2} v_{1}(x)}{d x^{2}}-C_{22} \frac{d^{2} v_{0}(x)}{d x^{2}}-x C_{21} u_{1}^{\prime}(x)-x C_{22} u_{0}^{\prime}(x)-C_{21} u_{0}(x) v_{1}(x)-C_{21} u_{1}(x) v_{0}(x) \\
& -C_{22} u_{0}(x) v_{0}(x)+C_{22}\left(x^{5}-x^{4}+2 x^{3}+x^{2}-x+2\right)=0, \\
& v_{2}(0)=0 \text {, } \\
& v_{2}(1)=0 \text {, } \\
& \Downarrow \\
& v_{2}(x)=\frac{1}{29566517460480000}(-1+x) x\left(-5320\left(-3060\left(15492027+15492027 x+282879147 x^{2}+148230633 x^{3}\right.\right.\right. \\
& -43985391 x^{4}+48434937 x^{5}-9694563 x^{6}+5614302 x^{7}+807500 x^{8}-337644 x^{9}+419112 x^{10}-53724 x^{11} \\
& \left.+11880 x^{12}\right)+\left(-2449166800-2449166800 x+87543536000 x^{2}+42225782090 x^{3}+86425737890 x^{4}\right. \\
& -43926601942 x^{5}+31708371758 x^{6}-1629973282 x^{7}+1692595968 x^{8}+1734798128 x^{9}-461535232 x^{10} \\
& \left.\left.+374063558 x^{11}-51230542 x^{12}+8414978 x^{13}+11711414 x^{14}-2313025 x^{15}+1001000 x^{16}\right) C_{11}\right) C_{21} \\
& +\left(288103926363274+288103926363274 x+4640952330267274 x^{2}+2430417680443564 x^{3}\right. \\
& -698705417457236 x^{4}+686062976647804 x^{5}-215138606473076 x^{6}+161390272754209 x^{7}+24160972706369 x^{8} \\
& -13483860439551 x^{9}+14256523191045 x^{10}-3171104413615 x^{11}+1093200643705 x^{12}-78130936175 x^{13} \\
& \left.-13083538539 x^{14}+30960042812 x^{15}-7686610408 x^{16}+2008972552 x^{17}-202323528 x^{18}+23023440 x^{19}\right) C_{21}^{2} \\
& +16279200\left(15492027+15492027 x+282879147 x^{2}+148230633 x^{3}-43985391 x^{4}+48434937 x^{5}-9694563 x^{6}\right. \\
& \left.\left.+5614302 x^{7}+807500 x^{8}-337644 x^{9}+419112 x^{10}-53724 x^{11}+11880 x^{12}\right) C_{22}\right) \text {. }
\end{aligned}
$$

By using (10), (e) part of the OHAM algorithm, solutions of the system containing auxiliary constants are

$$
\begin{aligned}
& u(x)=u_{0}(x)+u_{1}(x)+u_{2}(x)+\cdots, \\
& v(x)=v_{0}(x)+v_{1}(x)+v_{2}(x)+\cdots
\end{aligned}
$$

Using (f) and (g) of OHAM algorithm, one can get the auxiliary constants for $u(x)$

$$
\begin{aligned}
& C_{11}=-1.0735232305739808 ; \\
& C_{12}=-0.006525944134731277
\end{aligned}
$$

and the auxiliary constants for $v(x)$

$$
\begin{aligned}
& C_{21}=-0.9248766114149304 ; \\
& C_{22}=-0.041471006594628074 .
\end{aligned}
$$

By using auxiliary constants given in (28) and (29), the solutions of system $u(x)$ and $v(x)$, respectively, are

$$
\begin{aligned}
& u(x)=x(-1.000059796120042 \\
& +0.9996416248662816 x^{2} \\
& +0.00018671311678283842 x^{3}
\end{aligned}
$$




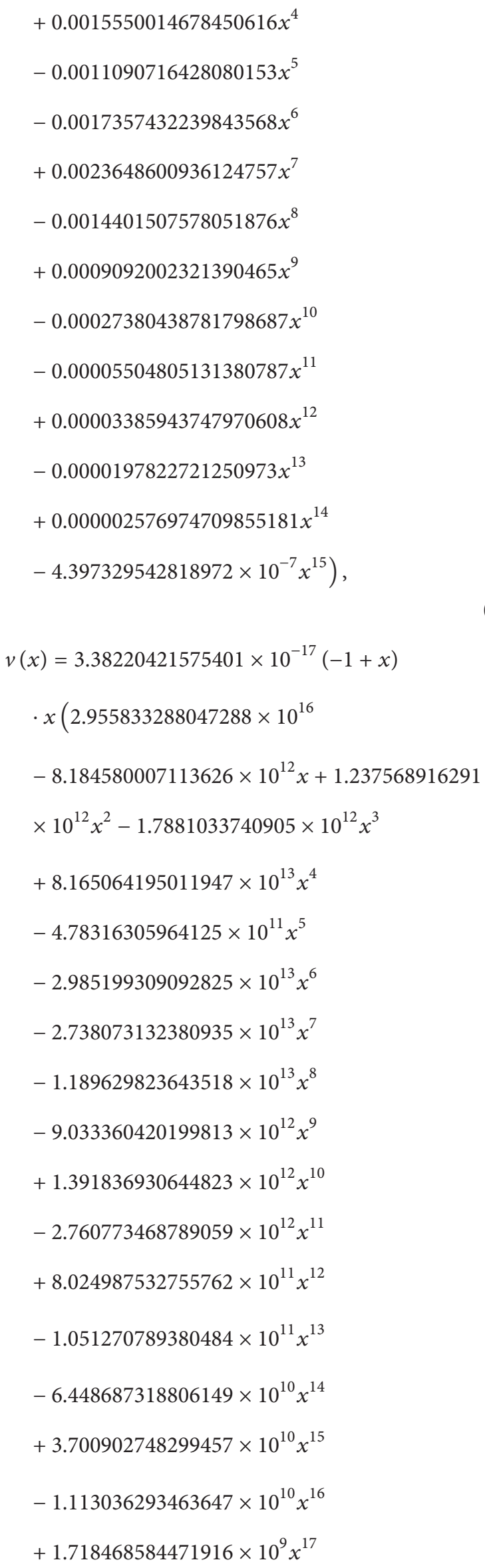

$$
\begin{aligned}
& -1.730668875597082 \times 10^{8} x^{18} \\
& \left.+1.969417566560864 \times 10^{7} x^{19}\right) .
\end{aligned}
$$

$3 r d$ Choice $\left(f_{11}(x)=-2 x^{2}+6 x\right.$ and $\left.f_{21}(x)=-x+2\right)$. The expressions for zeroth-order, first-order, and second-order problems of the system and their solutions are given below:

$$
\begin{aligned}
& \frac{d^{2} u_{0}(x)}{d x^{2}}+2 x^{2}-6 x=0, \\
& u_{0}(0)=0 \text {, } \\
& u_{0}(1)=0 \text {, } \\
& \Downarrow \\
& u_{0}(x)=\frac{1}{6}\left(-5 x+6 x^{3}-x^{4}\right) ; \\
& \frac{d^{2} v_{0}(x)}{d x^{2}}+x-2=0, \\
& v_{0}(0)=0 \text {, } \\
& v_{0}(1)=0 \text {, } \\
& \Downarrow \\
& v_{0}(x)=\frac{1}{6}\left(-5 x+6 x^{2}-x^{3}\right) \\
& \frac{d^{2} u_{1}(x)}{d x^{2}}-\left(1+C_{11}\right) \frac{d^{2} u_{0}(x)}{d x^{2}}+x C_{11} v_{0}^{\prime}(x) \\
& -C_{11} u_{0}(x)+C_{11}\left(x^{3}-2 x^{2}+6 x\right)-2 x^{2}+6 x \\
& =0 \text {, } \\
& u_{1}(0)=0 \text {, } \\
& u_{1}(1)=0 \text {, } \\
& \Downarrow \\
& u_{1}(x)=\frac{1}{360} x\left(53-60 x^{3}+9 x^{4}-2 x^{5}\right) C_{11} ; \\
& \frac{d^{2} v_{1}(x)}{d x^{2}}-\left(1+C_{21}\right) \frac{d^{2} v_{0}(x)}{d x^{2}}-x C_{21} u_{0}^{\prime}(x) \\
& -C_{21} u_{0}(x) v_{0}(x)+C_{21}\left(x^{5}-x^{4}+2 x^{3}+x^{2}-x\right. \\
& +2)-x+2=0 \text {, } \\
& v_{1}(0)=0 \text {, } \\
& v_{1}(1)=0 \text {, } \\
& \Downarrow \\
& v_{1}(x)=\frac{1}{90720} x\left(15451-12600 x^{2}-2310 x^{3}\right.
\end{aligned}
$$




$$
\begin{aligned}
& \left.+756 x^{4}-1092 x^{5}+300 x^{6}-540 x^{7}+35 x^{8}\right) C_{21} ; \\
& \frac{d^{2} u_{2}(x)}{d x^{2}}-\left(1+C_{11}\right) \frac{d^{2} u_{1}(x)}{d x^{2}}-C_{12} \frac{d^{2} u_{0}(x)}{d x^{2}} \\
& +x C_{11} v_{1}^{\prime}(x)+x C_{12} v_{0}^{\prime}(x)-C_{11} u_{1}(x) \\
& -C_{12} u_{0}(x)+C_{12}\left(x^{3}-2 x^{2}+6 x\right)=0 \text {, } \\
& u_{2}(0)=0 \text {, } \\
& u_{2}(1)=0 \text {, } \\
& \Downarrow \\
& u_{2}(x)=-\frac{1}{5987520} x\left(1 6 6 3 2 \left(-53+60 x^{3}-9 x^{4}\right.\right. \\
& \left.+2 x^{5}\right) C_{11}+\left(-786931+23045 x^{2}+997920 x^{3}\right. \\
& -274428 x^{4}+46200 x^{5}+2376 x^{6}-7128 x^{7} \\
& \left.+1925 x^{8}-3168 x^{9}+189 x^{10}\right) C_{11}^{2}+16632(-53 \\
& \left.\left.+60 x^{3}-9 x^{4}+2 x^{5}\right) C_{12}\right) ; \\
& \frac{d^{2} v_{2}(x)}{d x^{2}}-\left(1+C_{21}\right) \frac{d^{2} v_{1}(x)}{d x^{2}}-C_{22} \frac{d^{2} v_{0}(x)}{d x^{2}} \\
& -x C_{21} u_{1}^{\prime}(x)-x C_{22} u_{0}^{\prime}(x)-C_{21} u_{0}(x) v_{1}(x) \\
& -C_{21} u_{1}(x) v_{0}(x)-C_{22} u_{0}(x) v_{0}(x)+C_{22}\left(x^{5}\right. \\
& \left.-x^{4}+2 x^{3}+x^{2}-x+2\right)=0 \\
& v_{2}(0)=0, \\
& v_{2}(1)=0 \text {, } \\
& \Downarrow \\
& v_{2}(x)=\frac{1}{32691859200} x\left(3 6 0 3 6 0 \left(15451-12600 x^{2}\right.\right. \\
& -2310 x^{3}+756 x^{4}-1092 x^{5}+300 x^{6}-540 x^{7} \\
& \left.+35 x^{8}\right) C_{21}+\left(5674260280-3738374640 x^{2}\right. \\
& -1553326775 x^{3}+513080568 x^{4}-835014180 x^{5} \\
& +307934770 x^{6}-408648240 x^{7}+42182140 x^{8} \\
& +36036 x^{9}-2240784 x^{10}+1236235 x^{11} \\
& \left.-1362900 x^{12}+247500 x^{13}-10010 x^{14}\right) C_{21}^{2} \\
& +360360\left(15451-12600 x^{2}-2310 x^{3}+756 x^{4}\right. \\
& \left.\left.-1092 x^{5}+300 x^{6}-540 x^{7}+35 x^{8}\right) C_{22}\right) \text {. }
\end{aligned}
$$

By using (10), (e) part of the OHAM algorithm, solutions of the system containing auxiliary constants are

$$
\begin{aligned}
& u(x)=u_{0}(x)+u_{1}(x)+u_{2}(x)+\cdots, \\
& v(x)=v_{0}(x)+v_{1}(x)+v_{2}(x)+\cdots .
\end{aligned}
$$

Using (f) and (g) of OHAM algorithm, the auxiliary constants for $u(x)$ are

$$
\begin{aligned}
& C_{11}=-0.8635600588156002 ; \\
& C_{12}=-0.07024196528240835 ;
\end{aligned}
$$

and the auxiliary constants for $v(x)$ are

$$
\begin{aligned}
& C_{21}=-1.1001993642371848 ; \\
& C_{22}=-0.016142075251521698 ;
\end{aligned}
$$

By using auxiliary constants given in (34) and (35), the solutions of system $u(x)$ and $v(x)$, respectively, are as follows:

$$
\begin{aligned}
u(x) & \\
& =-0.000023539645681237463(-16.277938135162394 \\
& +x)(-0.99999999999999999+x) \\
& \cdot x(1.014839887401758+x)(2.677002513163501+x) \\
& \cdot(9.775577912415333-5.488002396636638 x) \\
& +x^{2}(10.514888024561234-1.3839014599187789 x \\
& \left.+x^{2}\right)(9.344985088509072+3.6960948292477878 x \\
& \left.+x^{2}\right), \\
v & (x)=-3.706271559217327 \\
& \times 10^{-7}(-17.17233411753372+x) \\
& +(-6.831733583228521+x)(-2.605228511492559 \\
& +x)(-1+x) x(6.323285413907099 \\
& \left.-4.0948461923897135 x+x^{2}\right)(6.460657989776115 \\
& \left.-1.7104472025776825 x+x^{2}\right)(6.727957744564565 \\
& \left.+1.0578696024575174 x+x^{2}\right)(5.635709976647391 \\
& \left.+2.9634757469789625 x+x^{2}\right)(5.703364669216431 \\
& \left.+4.667969532510989 x+x^{2}\right) .
\end{aligned}
$$

It has been observed that exact solutions are obtained by the proper choices of $f_{i 1}(x)$ in all three examples. In Tables 1 and 2, approximate solutions $u(x)$ and $v(x)$ are reliable for systems of boundary value problems having different choices of $f_{i 1}(x)$. Absolute errors of 2 nd-order solutions using OHAM are in excellent agreement with 9th-order solutions of HPM as shown in Tables 1 and 2. Therefore, extended formulation is very trustworthy for solutions of systems of boundary value problems. 
TABLE 1: Absolute errors of 2nd-order solutions of $u(x)$ for different choices of $f_{i 1}(x)$ and also comparison with the 9th-order HPM solutions.

\begin{tabular}{|c|c|c|c|c|c|}
\hline \multirow{2}{*}{$x$} & \multirow{2}{*}{ Exact } & \multirow{2}{*}{$\begin{array}{c}\mid \text { Exact }-u_{9}(x) \mid \\
\text { HPM [3] }\end{array}$} & \multicolumn{3}{|c|}{$\mid$ Exact $-u_{2}(x) \mid$} \\
\hline & & & 1st choice & 2nd choice & 3rd choice \\
\hline 0.1 & -0.099 & $8.5 \times 10^{-6}$ & 0.0 & $6.30503 \times 10^{-6}$ & $4.48845 \times 10^{-6}$ \\
\hline 0.2 & -0.192 & $1.6 \times 10^{-5}$ & 0.0 & $1.41177 \times 10^{-5}$ & $8.36115 \times 10^{-7}$ \\
\hline 0.3 & -0.273 & $2.3 \times 10^{-5}$ & 0.0 & $2.33804 \times 10^{-5}$ & $1.10537 \times 10^{-5}$ \\
\hline 0.4 & -0.336 & $2.9 \times 10^{-5}$ & 0.0 & $3.22826 \times 10^{-5}$ & $2.97367 \times 10^{-5}$ \\
\hline 0.5 & -0.375 & $3.3 \times 10^{-5}$ & 0.0 & $3.81526 \times 10^{-5}$ & $5.6901 \times 10^{-5}$ \\
\hline 0.6 & -0.384 & $3.4 \times 10^{-5}$ & 0.0 & $3.86649 \times 10^{-5}$ & $9.75798 \times 10^{-5}$ \\
\hline 0.7 & -0.357 & $3.3 \times 10^{-5}$ & 0.0 & $3.41025 \times 10^{-5}$ & $1.54265 \times 10^{-4}$ \\
\hline 0.8 & -0.288 & $2.7 \times 10^{-5}$ & 0.0 & $2.51968 \times 10^{-5}$ & $2.12191 \times 10^{-4}$ \\
\hline 0.9 & -0.171 & $1.6 \times 10^{-5}$ & 0.0 & $1.38339 \times 10^{-5}$ & $2.11514 \times 10^{-4}$ \\
\hline
\end{tabular}

TABLE 2: Absolute errors of 2nd-order solutions of $v(x)$ for different choices of $f_{i 1}(x)$ and also comparison with the 9th-order HPM solutions.

\begin{tabular}{lccccc}
\hline$x$ & Exact & $\begin{array}{c}\mid \text { Exact }-v_{9}(x) \mid \\
\text { HPM [3] }\end{array}$ & 1st choice & \multicolumn{2}{c}{$\mid$ Exact $-v_{2}(x) \mid$} \\
2nd choice & $2.73481 \times 10^{-5}$ & $7.24002 \times 10^{-5}$ \\
\hline 0.1 & -0.09 & $3.9 \times 10^{-5}$ & 0.0 & $5.22651 \times 10^{-5}$ & $1.29739 \times 10^{-4}$ \\
0.2 & -0.16 & $7.8 \times 10^{-5}$ & 0.0 & $7.06381 \times 10^{-5}$ & $1.60122 \times 10^{-4}$ \\
0.3 & -0.21 & $1.2 \times 10^{-4}$ & 0.0 & $7.68443 \times 10^{-5}$ & $1.56816 \times 10^{-4}$ \\
0.4 & -0.24 & $1.6 \times 10^{-4}$ & 0.0 & $6.63508 \times 10^{-5}$ & $1.20279 \times 10^{-4}$ \\
0.5 & -0.25 & $2.0 \times 10^{-4}$ & 0.0 & $4.01111 \times 10^{-5}$ & $5.953 \times 10^{-5}$ \\
0.6 & -0.24 & $2.4 \times 10^{-4}$ & 0.0 & $8.66203 \times 10^{-6}$ & $7.8516 \times 10^{-6}$ \\
0.7 & -0.21 & $2.7 \times 10^{-4}$ & 0.0 & $8.7425 \times 10^{-6}$ & $5.7654 \times 10^{-5}$ \\
0.8 & -0.16 & $2.9 \times 10^{-4}$ & 0.0 & $1.01876 \times 10^{-6}$ & $6.30638 \times 10^{-5}$ \\
0.9 & -0.09 & $2.4 \times 10^{-4}$ & 0.0 & &
\end{tabular}

\section{Conclusions}

In this paper, it has been revealed that the optimal homotopy asymptotic method can be applied effectively for solving the systems of second-order boundary value problems. This method is straightforward and easy to practice for solving the problems without any requirement of discretization of the variables. Therefore, it is not exaggerated by computational round of errors. As an advantage of the generalized optimal homotopy asymptotic method over the other procedures, it delivers the exact solutions of the problems depending upon the selection of $f_{i 1}(x)$. Moreover, the proposed method is free from rounding off errors and does not require excessive computer power or memory for its implementation.

\section{Competing Interests}

The authors declare that they have no competing interests.

\section{References}

[1] F. Geng and M. Cui, "Solving a nonlinear system of second order boundary value problems," Journal of Mathematical Analysis and Applications, vol. 327, no. 2, pp. 1167-1181, 2007.

[2] J. Lu, "Variational iteration method for solving a nonlinear system of second-order boundary value problems," Computers and Mathematics with Applications, vol. 54, no. 7-8, pp. 1133$1138,2007$.
[3] A. Saadatmandi, M. Dehghan, and A. Eftekhari, "Application of He's homotopy perturbation method for non-linear system of second-order boundary value problems," Nonlinear Analysis. Real World Applications, vol. 10, no. 3, pp. 1912-1922, 2009.

[4] A. Saadatmandi and J. A. Farsangi, "Chebyshev finite difference method for a nonlinear system of second-order boundary value problems," Applied Mathematics and Computation, vol. 192, no. 2, pp. 586-591, 2007.

[5] D.-H. Shou and J.-H. He, "Application of parameter-expanding method to strongly nonlinear oscillators," International Journal of Nonlinear Sciences and Numerical Simulation, vol. 8, no. 1, pp. 121-124, 2007.

[6] M. Dehghan and M. Lakestani, "Numerical solution of nonlinear system of second-order boundary value problems using cubic B-spline scaling functions," International Journal of Computer Mathematics, vol. 85, no. 9, pp. 1455-1461, 2008.

[7] N. Herişanu, V. Marinca, T. Dordea, and G. Madescu, "A new analytical approach to nonlinear vibration of an electrical machine," Proceedings of the Romanian Academy, Series A, vol. 9, no. 3, pp. 229-236, 2008.

[8] V. Marinca and N. Herişanu, "Application of optimal homotopy asymptotic method for solving nonlinear equations arising in heat transfer," International Communications in Heat and Mass Transfer, vol. 35, no. 6, pp. 710-715, 2008.

[9] V. Marinca, N. Herişanu, C. Bota, and B. Marinca, "An optimal homotopy asymptotic method applied to the steady flow of a fourth-grade fluid past a porous plate," Applied Mathematics Letters, vol. 22, no. 2, pp. 245-251, 2009. 
[10] V. Marinca, N. Herişanu, and I. Nemeş, "Optimal homotopy asymptotic method with application to thin film flow," Central European Journal of Physics, vol. 6, no. 3, pp. 648-653, 2008.

[11] S. Iqbal, M. Idrees, A. M. Siddiqui, and A. Ansari, "Some solutions of the linear and nonlinear Klein-Gordon equations using the optimal homotopy asymptotic method," Applied Mathematics and Computation, vol. 216, no. 10, pp. 2898-2909, 2010.

[12] S. Iqbal and A. Javed, "Application of optimal homotopy asymptotic method for the analytic solution of singular LaneEmden type equation," Applied Mathematics and Computation, vol. 217, no. 19, pp. 7753-7761, 2011.

[13] M. S. Hashmi, N. Khan, and S. Iqbal, "Numerical solutions of weakly singular Volterra integral equations using the optimal homotopy asymptotic method," Computers \& Mathematics with Applications, vol. 64, no. 6, pp. 1567-1574, 2012.

[14] M. S. Hashmi, N. Khan, and S. Iqbal, "Optimal homotopy asymptotic method for solving nonlinear Fredholm integral equations of second kind," Applied Mathematics and Computation, vol. 218, no. 22, pp. 10982-10989, 2012.

[15] A. Zeb, S. Iqbal, A. M. Siddiqui, and T. Haroon, "Application of the optimal homotopy asymptotic method to flow with heat transfer of a pseudoplastic fluid inside a circular pipe," Journal of the Chinese Institute of Engineers, vol. 36, no. 6, pp. 797-805, 2013.

[16] S. Iqbal, A. R. Ansari, A. M. Siddiqui, and A. Javed, "Use of optimal homotopy asymptotic method and galerkin's finite element formulation in the study of heat transfer flow of a third grade fluid between parallel plates," Journal of Heat Transfer, vol. 133, no. 9, Article ID 091702, 2011.

[17] N. Herisanu, V. Marinca, and G. Madescu, "An analytical approach to non-linear dynamical model of a permanent magnet synchronous generator," Wind Energy, vol. 18, no. 9, pp. 1657-1670, 2015.

[18] N. Herisanu and V. Marinca, "Optimal homotopy perturbation method for non-conservative dynamical system of a rotating electrical machine," Zeitschrift fur Naturforschung A, vol. 67, pp. 509-516, 2012. 


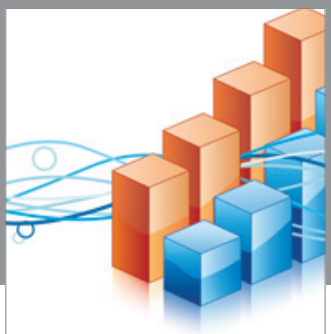

Advances in

Operations Research

vatem alat4

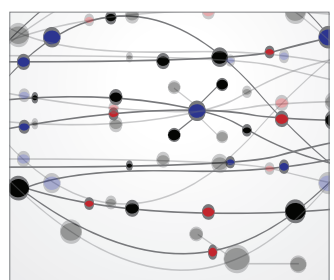

\section{The Scientific} World Journal
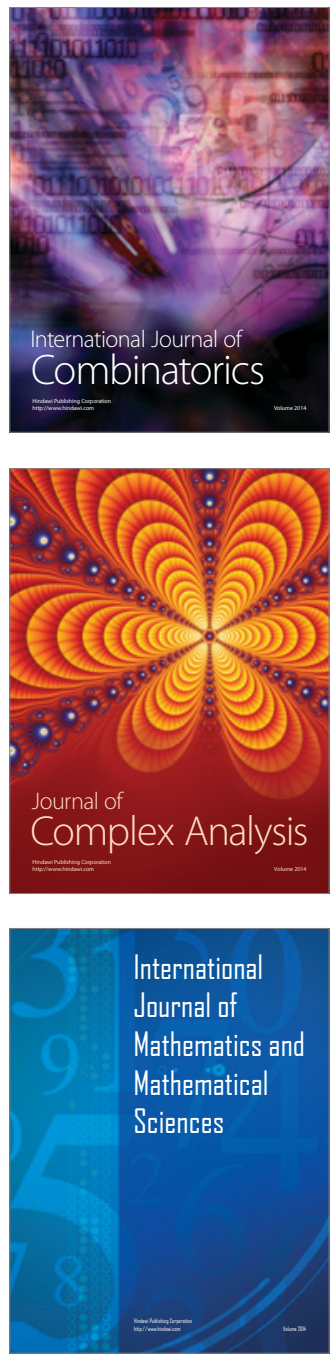
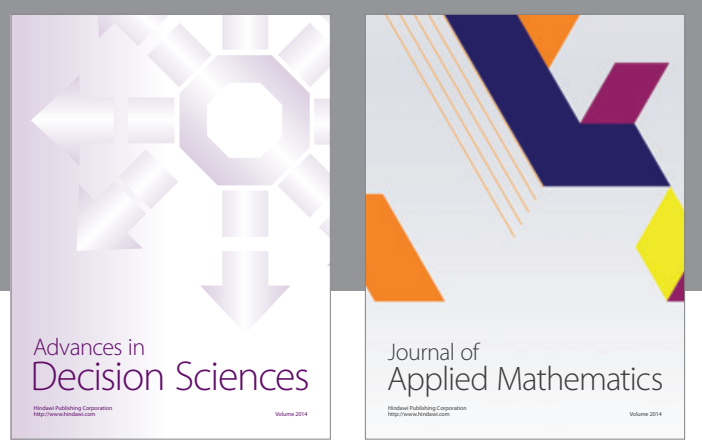

Algebra

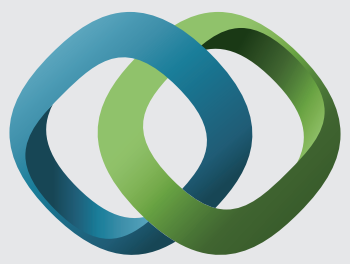

\section{Hindawi}

Submit your manuscripts at

https://www.hindawi.com
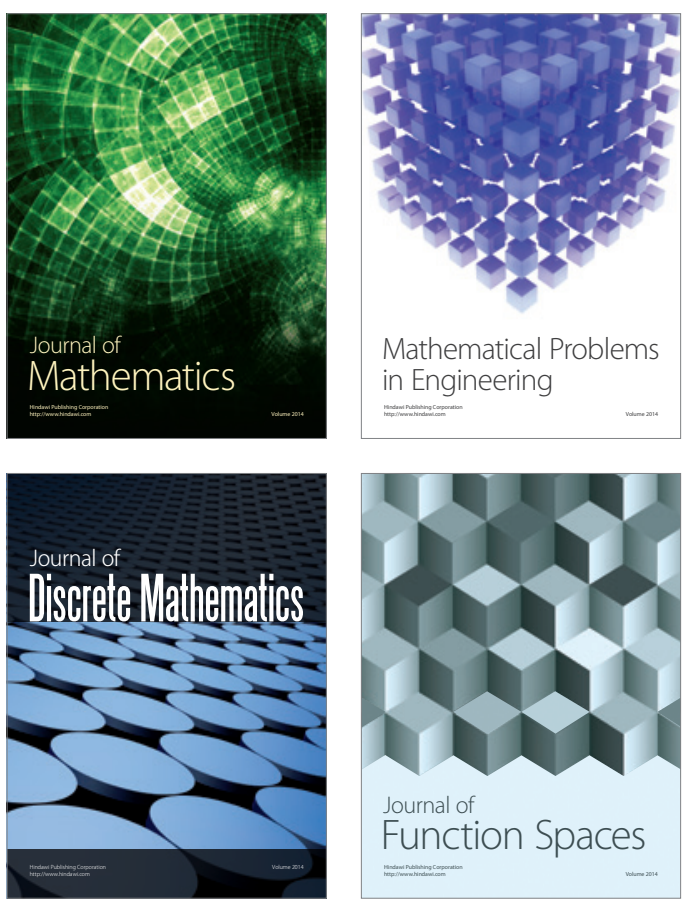

Mathematical Problems in Engineering
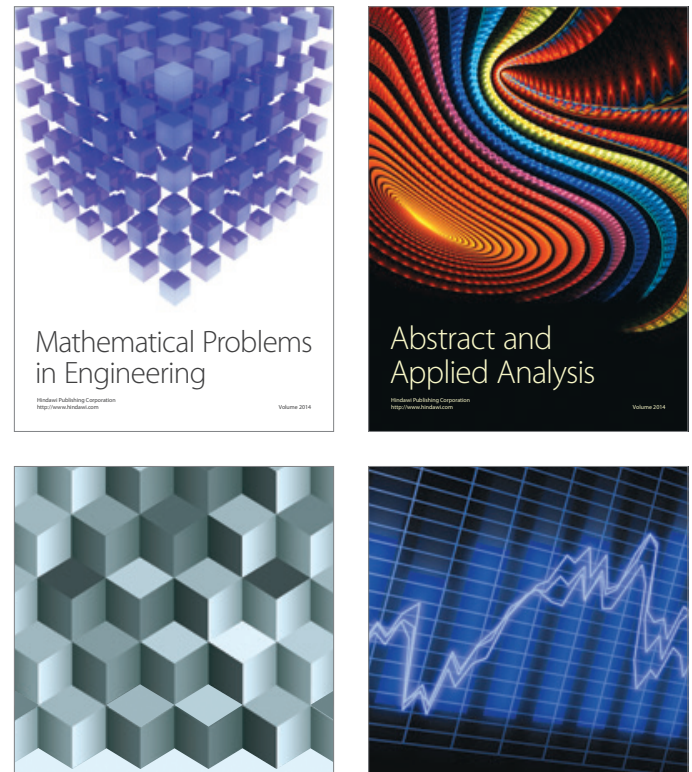

Journal of

Function Spaces

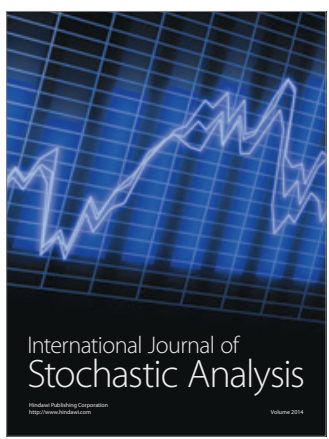

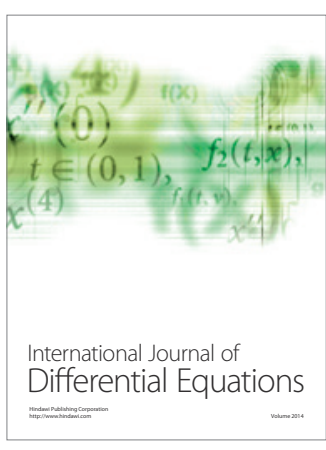
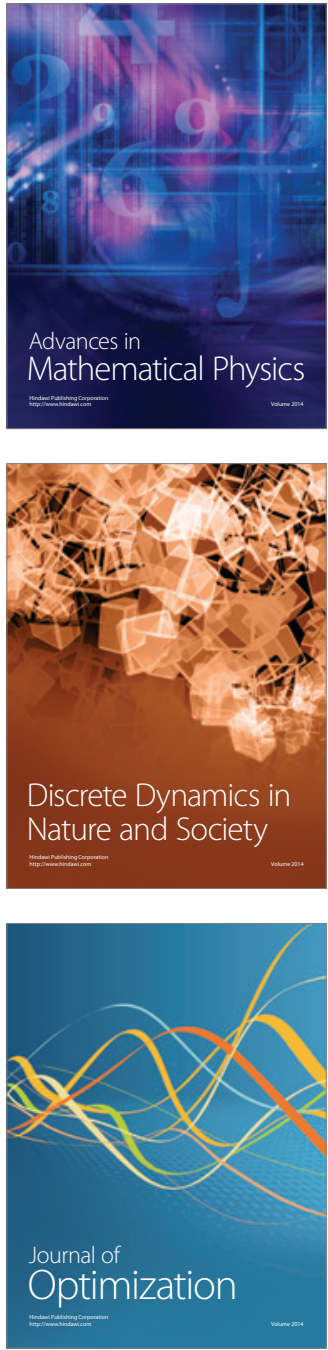